\title{
Open Surgery for Urinary Stones in a Resource Poor Setting: A Look at Dalhatu Araf Specialist Hospital, Lafia, Nigeria
}

\author{
Christian A. Agbo ${ }^{凶}$ \\ Institution-Dalhatu Araf Specialist Hospital, Lafia, Nigeria \\ Soc Int Urol J.2021;2(2):79-81 \\ DOI: https://10.48083/KF0Z6048
}

\section{Introduction}

Urinary stone disease has afflicted humankind since antiquity[1]. It remains a common urological condition worldwide, including in our environment[2]. Although open surgery was previously the main option for stone removal, advances in technology mean that treatment is now largely through minimally invasive surgery, as recommended by a number of urological guidelines $[3,4]$. Unfortunately, at our centre, we still treat urinary stones solely through open surgery, mostly because we lack endoscopic equipment. In addition, most of our patients, even if referred to facilities where endoscopic management is possible, cannot afford the cost of treatment.

\section{Dalhatu Araf Specialist Hospital}

The Dalhatu Araf Specialist Hospital is a tertiary multispecialty public hospital located in Lafia, a northern part of Nigeria, which, because of the climate, has a high incidence of urologic stones even in children as young as one year old. Within the state, which has a population of about 2 million, there is another tertiary institute (in Keffi) that has 2 urologists. Further afield, there are bigger hospitals in Abuja, Jos, and Lagos that can undertake other procedures. Some patients are referred to Abuja, but it is a 3 -hour drive from Lafia, and we can refer only those who can afford to pay-about $90 \%$ cannot.

\section{Management of Urinary Stones in Our Environment}

The Dalhatu Araf Specialist Hospital has X-ray and ultrasound equipment, and CT is available at a nearby centre. The hospital also has video equipment, but there is no funding for instruments. I have, therefore, purchased 2 cystoscopes, but they are rigid, rather than flexible, representing technology from the 1960s or 1970s, and they do not provide the same view as the more modern flexible scopes. The lack of technology is particularly frustrating because I spent 6 months in India training in the endoscopic management of stones, so I have the skill to undertake minimally invasive procedures if the equipment were available.

\section{TABLE 1}

Overview of the open surgery done for urinary stones seen at Dalhatu Araf specialist hospital, Lafia, Nigeria within a 2-year period

Location of stone

\begin{tabular}{|l|l|l}
\hline Single & 6 & 21.4
\end{tabular}

Renal

\begin{tabular}{l|l|l} 
Staghorn & 3 & 10.7
\end{tabular}

\begin{tabular}{l|c|c|c|}
\hline Ureter & 5 & 17.9 & Ureterolithotomy \\
\hline Bladder & 11 & 39.3 & Cystolithotomy \\
\hline Urethral & 3 & 10.7 & Urethrolithotomy \\
\hline Total & 28 & 100.0 & \\
\hline
\end{tabular}

\section{Types of open surgery}

Anatrophic nephrolithotomy

Extended pyelolithotomy

Anatrophic nephrolithotomy

Extended pyelolithotomy 


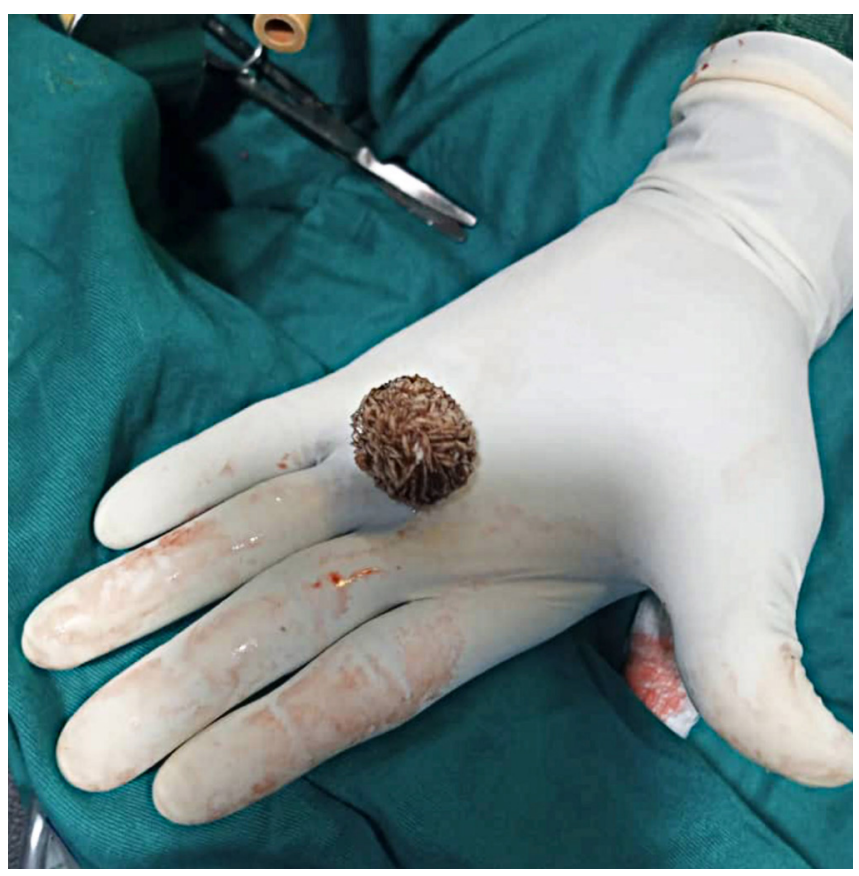

FIGURE 1.

A stone from the urinary bladder via open cystolithotomy

Urinary stones account for about $30 \%$ of my caseload as the hospital's single urologist. In the last 2 years, 28 open surgeries for urinary stones were undertaken at the hospital (Table 1), including anatrophic nephrolithotomy, extended pyelolithotomy, ureterolithotomy, cystolithotomy (Figure 1), and urethrolithotomy. Patients with urinary stones are referred here from other smaller centres within the state. A good proportion (55.4\%) of the patients are managed non-surgically, in accordance with the European Association of Urology guidelines. These are patients who are asymptomatic or non-obstructing and those that have medical expulsive therapy for lower ureteric stone.

The patients are financially responsible for their health care but at a very subsidized rate because of the low socioeconomic status of the people, many of whom are subsistence farmers, growing rice and yams. Cost is therefore a major consideration in treatment. If patients come in for surgery, they pay the hospital US $\$ 100$ for stone management (more if there are complications). For a lot of patients, this is more than one month's salary, and about $2 / 3$ of patients are still managed without surgery.

The commonest indication for surgery is persistent pain (61.2\%) followed by obstruction. All patients who meet the indication for surgery are managed by open surgeries. Although open surgery still has a role in stone management in cases of complex stone, Hippocrates discouraged open surgeries for stone. His oath reads:
'I will not cut persons labouring under the stone, but will leave this to be done by practitioners of this work' [5]. The majority of urinary stones can be managed endoscopically[6]. In the near future, we hope to manage stones endoscopically, which will require additional training and equipment.

When surgery is required, patients are admitted a day before the procedure. The average hospital stay is 5 days. Both external and internal stents are used here depending on the availability and affordability at time of surgery. When patients undergo pyelolithotomy, stents are usually put in, which can entail a hospital stay of up to 5 to 7 days (when external stent is used), depending on drainage. In some cases (when internal stent is used), they stay 2 to 3 days, and then come back for removal at 6 weeks post-procedure. When an external stent is used, it is passed through the kidney, as with nephrostomy. Instead of a single J stent, we use a feeding tube, which is far less expensive and works just as well.

As the sole urologist at the centre, I am also responsible for the patient's postoperative care, which can be quite challenging. Although we do have residents in general surgery, who assist me, as well as residents doing a three-month rotation in urology and subspecialties, there are no urology residents-and as the hospital does not have accreditation, we do not have visiting residents from Abuja or Lagos.

Postoperatively, the patient's family is responsible for a lot of the care, including provision and preparation of food. Pain medications are provided while the patient is in hospital, but at discharge, the patient assumes responsibility and must obtain needed drugs from the nurses or the pharmacy within the hospital.

The hospital also sees a lot of benign prostatic hyperplasia. Currently, it is not possible to do anything but retropubic simple prostatectomy because the hospital does not yet have the equipment to permit transurethral resection of prostate. We are looking forward to getting equipment. Many patients cannot afford medical therapy, so they undergo simple prostatectomy, which I do here (probably 2 a month). Postoperatively, patients are catheter-dependent for 7 to 10 days for transvesical prostatectomy, and most are comfortable with that.

Difficulty in getting to the centre or concerns about the cost of treatment means that patients with bladder cancer often present at an advanced stage. Once the diagnosis has been confirmed by cystoscopy, patients are referred to a higher-level centre for transurethral resection of bladder tumour. Chemotherapy is also available if patients can afford it. Otherwise, radical cystectomy is performed at our centre. Regardless, patients are often lost to follow-up. 


\section{Conclusion}

Although open surgery still has a role in complex stone disease and in patients with anatomical and physiological anomalies, the majority of urinary stones can be managed by minimally invasive surgeries. To date, attempts to secure funding (from government bodies and from private foundations) for equipment

\section{References}

1. Tefekli A, Cezayirli F. The history of urinary stones: in parallel with civilization. Scientific World Journal.2013;Article ID 423964. doi. org/10.1155/2013/423964

2. Çakici ÖU, Ener K, Keske M, Altinova S, Canda AE, Aldemir M, et al. Open stone surgery: a still-in-use approach for complex stone burden. Cent European J Urol.2017;70(2):179-184.

3. Tzelves L, Türk C, Skolarikos A. European Association of Urology Urolithiasis Guidelines: Where Are We Going? Eur Urol Focus. 2020;S2405-4569(20):30270-30274. doi: 10.1016/j.euf.2020.09.011. that would allow stones to be managed endoscopically have been unsuccessful. We continue to seek support and funding for training and provision of endoscopic equipment that will enable us to meet the global standard of care.

4. Zumstein V, Betschart P, Abt D, Schmid HP, Panje CM, Putora PM. Surgical management of urolithiasis - a systematic analysis of available guidelines. BMC Urol.2018 Apr 10;18(1):25.

5. Buchholz N, Elhowairis ME, Bach C, Moraitis K, Masood J. From 'stone cutting' to high-technology methods: The changing face of stone surgery. Arab J Urol.2011 Mar;9(1):25-27.

6. El-Husseiny T, Buchholz N. The role of open stone surgery. Arab J Urol.2012;10(3):284-288. 